HNO 2009 • 57:196-198

DOI 10.1007/s00106-008-1854-3

Online publiziert: 28. Februar 2009

(c) Springer Medizin Verlag 2009

\author{
M. Tisch \\ Klinik und Poliklinik für HNO-Heilkunde, Kopf- und Halschirurgie, \\ Bundeswehrkrankenhaus, UIm
}

\title{
Hörrehabilitation mittels implantierbarer Hörsysteme
}

Hören zu können, ist von allen Sinneswahrnehmungen die wichtigste Voraussetzung für die geistige Entwicklung des Menschen und für ein sozial integrierendes Leben in der Gesellschaft. Rund 14 Mio. Menschen in Deutschland leiden unter mehr oder minder starker Schwerhörigkeit. Ohne Behandlung entstehen bei Kindern Sprachentwicklungsstörungen, bei Erwachsenen droht die soziale Isolation.

Als Therapie kam bis vor 15 Jahren ausschließlich eine konventionelle Hörgeräteversorgung in Betracht. Gerade bei einer hochgradigen Schwerhörigkeit stoßen jedoch konventionelle Hörgeräte aufgrund bauartbedingter technischer Einschränkungen an ihre Grenzen. Hier sind Hörgeräteimplantate aufgrund der Trennung ihrer Einzelkomponenten nach wie vor im Vorteil.

\section{() Bei hochgradiger Schwerhörigkeit sind Hörgeräteimplantate von Vorteil}

Sie bieten in der Regel eine bessere Klangqualität und differenziertere Spracherkennung dank geringerer Verzerrung. Daneben spielen auch medizinische Gründe, wie z. B. intolerable Okklusionen des Gehörgangs und rezidivierende Gehörgangsentzündungen bei der Entscheidung für ein Hörgeräteimplantat eine Rolle. Für eine Reihe von Patienten spielt auch die Stigmatisierung durch ein konventionelles Hörgerät, sei es der rein kosmetische Aspekt oder die tat- sächliche, zum Teil erhebliche Benachteiligung im Berufsleben eine wesentliche Rolle bei der Entscheidung für ein voll- oder teilimplantierbares Hörsystem. Auch die berufliche Situation (Arzt/Pflegepersonal - Stethoskop) oder nicht vermeidbare Arbeitsbedingungen (feuchte/heiße Umgebung) können die Verwendung konventioneller Hörgeräte erschweren oder unmöglich machen [8].

Mit Hilfe der Entwicklung voll- und teilimplantierbarer Hörsysteme, die als sog. vibratorische Verstärkerimplantate arbeiten, ist für viele Patienten zwischenzeitlich eine Lösung der oben skizzierten Probleme möglich. Vibratorische Verstärkerimplantate für mittel- bis hochgradige Innenohrschwerhörige produzieren mikromechanische Vibrationen. Im Gegensatz zu konventionellen Hörgeräten geben sie also keinen Schall ab, sondern arbeiten mit einem elektromechanischen Aktor, der den aufgenommenen Schall in Bewegungen umsetzt und im Sinne eines Direct-drive-Systems die Gehörknöchelchenkette direkt antreibt. Hieraus resultiert im Wesentlichen die deutlich bessere Verstärkungsleistung aktiver teil- und vollimplantierbarer Hörsysteme [8].

Implantate haben hierbei den großen Spagat zwischen möglichst perfektem Hören, hoher Zuverlässigkeit, geringer Ausfallrate, geringen operativen Risiken, überschaubaren Kosten und geringer Sichtbarkeit zu bewältigen.

Die Entwicklung der aktiven Mittelohrimplantate hat hierbei in den letzten 15 Jahren rasant zugenommen. Federführend für diese Entwicklungen war das ers- te in Deutschland zugelassene vollimplantierbare aktive Mittelohrimplantat TICA (Fa. Implex, München), aus der Arbeitsgruppe von Prof. Zenner. Sowohl die Technik als auch die Patientenzufriedenheit und die audiologischen Ergebnisse dieses (heute nicht mehr verfügbaren) Systems stellten bis heute Standards auf, an denen sich alle anderen am Markt befindlichen Systeme messen lassen müssen. In unserer Klinik wurde 2 Patienten nach der Zulassung ein aktives Mittelohrimplantat vom Typ TICA eingesetzt. Die postoperativen Hörergebnisse und die Zufriedenheit mit dem Vollimplantat beider Patienten waren hervorragend.

Noch am Markt verfügbar sind derzeit teil- und vollimplantierbare, ossikelgekoppelte Implantate der Fa. MED-EL, Starnberg, der Fa. Otologics, Heidelberg und der Fa. Envoy, Köln. Grundsätzlich gilt, dass aktive Mittelohrimplantate immer dann indiziert sind, wenn eine konservative Hörgeräteversorgung nicht $\mathrm{zu}$ einer ausreichenden Klangqualität, zu Rückkopplungen und zu Verzerrungen mit mangelhafter Sprachverständlichkeit führt.

In Ergänzung dazu steht bei voll implantierbaren Hörgeräten (früher: TICA, aktuell: Fa. Otologics, Heidelberg, Fa. Envoy, Köln) ein breiteres Indikationsspektrum zur Verfügung. Bei im Gehörgang implantiertem Sensor ist außerdem ein wichtiger Gewinn an Störschallunterdrückung (verbessertes Sprachverständnis, z. B. bei beruflichem Hintergrundlärm) und Schalllokalisation (erleichterte Separation von Stör- und Nutzschall) auf- 
Hier steht eine Anzeige.

算 Springer 
grund der natürlichen Richtcharakteristik des Außenohrs zu erwarten. Wird das Mikrophon nahe dem Ohr implantiert, ergeben sich vergleichbare Vorteile, allerdings können hier Kaugeräusche ein erhebliches akustisches Überlagerungsproblem darstellen.

Dennoch haben Vollimplantate im Vergleich zu Teilimplantaten eine ganze Reihe von Vorteilen im täglichen Leben (z. B. bei Regen, Schwitzen, Windgeräuschen, Schwimmen, Duschen) und v. a. bei der Wiederherstellung der Arbeitsfähigkeit in bestimmten Berufsgruppen (beispielsweise Lehrer, insbesondere Sportlehrer, Berufsköche, Sitzungsleiter, Außendienstmitarbeiter, Call-CenterMitarbeiter, Telefonisten). Nicht zuletzt besteht auch von vielen Patienten, bei denen die Entscheidung für oder gegen ein aktives Mittelohrimplantat ansteht, der primäre Wunsch nach einem Vollimplantat - gemäß dem Motto „wenn schon ein Hörgerät, dann aber auch so, dass man nichts mehr sieht“. Der soziale Rückzug aus der menschlichen Kommunikation sowie berufliche Diffamierung und Diskriminierung sind für den Schwerhörigen besonders beeinträchtigend. Daher ist eine erfolgreiche soziale Reintegration, sei sie beruflich oder privat, die eigentliche klinische „Messlatte“ [8].

Bis vor 3 Jahren wurden aktive Mittelohrimplantate ausschließlich bei Patienten eingesetzt, die aus medizinischen Gründen mit konventionellen Hörgeräten nicht zufriedenstellend rehabilitiert werden konnten. Patienten, bei denen eine Schalleitungs- oder eine kombinierte Hörstörung vorlag, konnten von dieser neuen Technologie nicht profitieren, da eine frei bewegliche Kette für eine optimale Energieübertragung an das Innenohr erforderlich war $[2,5]$.

In den letzten 3 Jahren wurde mit der Entwicklung der Rundfenster-vibroplasty-Technik zusätzlich die Versorgung von Schalleitungsschwerhörigkeiten und kombinierten Schwerhörigkeiten mit aktiven Mittelohrimplantaten erschlossen $[1,3,7]$. Bei Kindern mit Gehörgangs- und Ohrmuschelatresien kann die kosmetische Rehabilitation mit einer Hörgeräteimplantation verbunden werden $[4,7]$. Eine wichtige Indikation stellt die Versorgung von Patienten mit chro- nischer Mittelohrentzündung und kombinierter Schwerhörigkeit dar, bei denen eine zufriedenstellende operative Hörrehabilitation nicht erzielt und eine postoperative konventionelle Hörgeräteversorgung aus technischen Gründen nicht durchgeführt werden konnte. Darüber hinaus liegen erste positive Ergebnisse bei der Hörrehabilitation von Otosklerosepatienten vor, die mit einer alleinigen Stapesplastik nicht suffizient versorgt werden konnten [6].

Fazit: Die direkte Übertragung von Schall auf die Gehörknöchelchen des Mittelohrs bietet eine bessere Verstärkung als konventionelle Hörgeräte. Während früher die reine Schallempfindungsschwerhörigkeit die einzige Indikation für vollund teilimplantierbare Hörsysteme war, hat sich in den letzten 3 Jahren v. a. die kombinierte Schwerhörigkeit als wesentliche Indikation herausgestellt. Gerade bei dieser Indikation können aktive Mittelohrimplantate ihre Vorteile gegenüber konventionellen Hörgeräten ausspielen und durch ein deutliches Plus beim Hören zu wesentlich mehr Lebensqualität beitragen.

\section{( Die direkte Übertragung von Schall auf die Gehörknöchel- chen des Mittelohrs bietet eine bessere Verstärkung als konventionelle Hörgeräte}

Die Entwicklung halboffener und offener konventioneller Hörgeräte hat jedoch in den letzten Jahren einen so rasanten Verlauf genommen, dass im Bereich der Schallempfindungsschwerhörigkeit die Indikationen für teilimplantierbare Hörsysteme deutlich zurückgegangen sind.

Grundsätzlich gilt, dass bei Beginn der Schwerhörigkeit konventionelle Hörgeräte die erste Wahl bleiben. Erst wenn der Patient damit nicht mehr ausreichend hört oder der Einsatz eines Hörgeräts aufgrund von Unverträglichkeiten oder Operationen des Mittelohrs nicht möglich/ sinnvoll ist, muss geprüft werden, ob ein aktives Mittelohrimplantat indiziert ist. In diesen Fällen sind Implantate eine wertvolle und inzwischen bewährte Ergänzung in der Versorgung fortgeschrittener Innenohr- und kombinierter Schwerhörigkeiten.

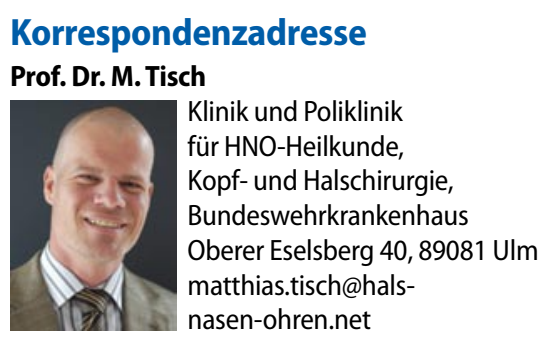

Interessenkonflikt. Keine Angaben.

\section{Literatur}

1. Colletti V, Soli S, Carner M, Colletti L (2006) Treatment of mixed hearing losses via implantation of a vibratory transducer on the round window. Int J Audiol 45(10): 600-608

2. Dazert S, Shehata-Dieler WE, Dieler R, Helms J (2000) Das Mittelohrimplantat „Vibrant Soundbridge" zur Hörrehabilitation bei sensorischer Schwerhörigkeit: Klinik, Indikation und erste Ergebnisse. Laryngorhinootologie 79(8): 459-464

3. Huber A, Ball G, Veraguth D et al (2006) A new implantable middle ear hearing device for mixed hearing loss: A feasibility study in human temporal. Otol Neurotol 27(8): 1104-1109

4. Kiefer J, Arnold W, Staudenmaier R (2006) Round window stimulation with an implantable hearing aid (Soundbridge ${ }^{\circledast}$ ) combined with autogenous reconstruction of the auricle - a new approach. ORL 68(6): 378-385

5. Truy E, Philibert B, Vesson JF et al (2008) Vibrant Soundbridge versus conventional hearing aids in sensorineural high-frequency hearing loss: A Prospective Study. Otol Neurotol 29(5): 684-687

6. Venail F, Lavieille JP, Meller R et al (2007) New perspectives for middle ear implants: First results in otosclerosis with mixed hearing loss. Laryngoscope 117(3): 552-555

7. Wollenberg B, Beltrame M, Schönweiler R et al (2007) Integration des aktiven Mittelohrimplantates in die plastische Ohrmuschelrekonstruktion. HNO 55(5): 349-356

8. Zenner HP (2001) Innenohrschwerhörigkeit: Elektronische Hörimplantate zur operativen Behandlung. Dtsch Ärztebl 98(4): 169-174 